\title{
Infrared Imaging of Capella with the IOTA Interferometer
}

\author{
Stefan Kraus $^{a b}$ and F. Peter Schloerb ${ }^{a}$ \\ ${ }^{a}$ Department of Astronomy, University of Massachusetts, Amherst MA 01003 \\ ${ }^{b}$ Max-Planck Insitut für Radioastronomie (MPIfR), Auf dem Hügel 69, D-53121 Bonn, \\ Germany
}

\begin{abstract}
We present near infrared aperture synthesis maps of the well known binary star Capella ( $\alpha$ Aur) produced with the upgraded IOTA interferometer on top of Mount Hopkins, Arizona. Michelson interferograms were obtained simultaneously on three interferometer baselines in the $H$-band between 2002 November 12 and 16 . The simultaneous observation of fringes on three baselines permitted a single phase closure to be estimated along with three visibility amplitudes. Hybrid Mapping techniques were then used to reconstruct the source brightness distribution with a beam size of $(5.4 \times 2.6)$ mas, which allows for the resolution of the stellar surfaces of the Capella giants. The maps provide the first demonstration of imaging with phase closure on the IOTA instrument.
\end{abstract}

Keywords: IOTA, Capella, Imaging

\section{INTRODUCTION}

The Infrared Optical Telescope Array (IOTA) is a long baseline optical interferometer which has been jointly constructed by the Smithsonian Astrophysical Observatory (SAO), Harvard University, the University of Massachusetts (UMass), the University of Wyoming, and MIT/Lincoln Laboratory. ${ }^{1}$ IOTA is located at the Fred Whipple Observatory atop Mount Hopkins, Arizona. Between 2000 and 2002, the interferometer was upgraded from two telescopes to a three-telescope system for the purpose of conducting research on interferometric imaging in the optical and near-ir. This upgrade was completed in early 2002, and IOTA demonstrated its first closure phases on 2002 February 25.

In this paper, we present initial results of a campaign of observations designed to produce an aperture synthesis map of the well known binary star Capella. This map was made for the purpose of demonstrating the ability of IOTA to measure and calibrate valid phase closure data and to explore different algorithms for the estimation of visibility amplitudes and closure phases from IOTA data. First results were published in the M.S. Thesis of S. Kraus. ${ }^{2}$ A scientific paper summarizing the complete work is in preparation by the IOTA team.

\section{OBSERVATIONS}

IOTA's telescopes can be moved on an L-shaped track and are mounted on stations $\approx 5 \mathrm{~m}$ (on the south-east baseline, $15 \mathrm{~m}$ long) and $\approx 7 \mathrm{~m}$ (on the north-east baseline, $35 \mathrm{~m} \mathrm{long}$ ) apart. The ability to move the telescope to several positions allowed a reasonable sampling of the UV plane to be carried out on Capella within a few days of observations. The data presented in this paper were obtained at the IOTA between UT 2002 November 12 and 16.

The optics at IOTA consist of $\mathrm{f} / 2.545 \mathrm{~cm}$ Cassegrain primary mirrors, which are fed by siderostats. The atmospherically induced motion of the image is compensated by tip-tilt adaptive optics mounted behind the telescopes. Path length compensation is carried out using two optical delay lines which continuously track the geometrical delay imposed by the orientation of the source with respect to the interferometer baselines. The data presented in this paper were obtained using the near-IR IONIC3 beam combiner. ${ }^{3}$ The beam combiner produces two complementary outputs for each of the three interferometer baselines. These outputs are detected

Further author information: (Send correspondence to F.P.S.)

F.P.S.: E-mail: schloerb@astro.umass.edu; S.K.: E-mail: skraus@mpifr-bonn.mpg.de 
by IOTA's PICNIC-camera. ${ }^{4}$ Fringes are produced by using piezo scanners installed in the beam path for two telescopes. The scanners continually modulate the geometric path delay in order to create interference fringes.

Raw fringe measurements are reduced to visibility measurements by a sequence of steps. First, we identify the interference fringe within each scan, which permits estimation of the zero optical path delay (OPD). A photometric correction is then performed for each of the 6 individual scans to remove the background and account for differences in the light transfer through the system. ${ }^{5}$ The complementary outputs of the beam combiner for each baseline are then differenced to produce the final scan through the white light fringe produced by the system. The amplitude of the fringe envelope is then used to estimate the raw visibility amplitude of the target star. Several techniques have been investigated for making this estimate, including use of wavelet transform methods, and all lead to consistent results for Capella. Typically the results of hundreds of individual fringe visibility estimates are obtained in a matter of minutes and averaged to produce a final estimate. All target observations are surrounded by measurements of unresolved calibration stars. Thus, the raw visibility amplitudes may be corrected for the transfer function of the instrument by making the ratio of the target visibility to the calibrator visibility.

The simultaneous measurements of three interference fringes permits the possibility to determine a closure phase for the three interferometer baselines. The phases of the three fringes, $\phi_{A B}, \phi_{B C}$, and $\phi_{A C}$, were estimated by locating the center of the fringe envelope for each baseline and setting a temporal window around the median time for all three fringes. This ensures that we sample within the same time interval, to assure phase closure, and it also means that the duration of the data used for the phase closure estimate is smaller than the coherence time of the atmosphere. The Fourier Transform of each scan is computed, and the fringe is located in the FT by finding the peak corresponding to the fringe frequency. In the IOTA three-baseline system, the frequencies of the three fringes obey a closure relationship similar to phase closure. Thus the peak frequency positions may be checked for consistency by requiring them to satisfy this relationship. If the frequency closure relationship is satisfied, then the phases of the fringes at the peak positions are used to compute the closure phase, $\Phi$, according to the definition: $\Phi=\phi_{A B}+\phi_{B C}-\phi_{A C}$. As in the case of the visibility amplitudes themselves, hundreds of individual measurements of closure phase, obtained over a time period of just a few minutes, are averaged together to achieve a final result. Unresolved calibrators are used to define the instrumental closure phase offset within the instrument. We find that the closure phase measured on calibrators throughout the night is quite consistent.

\section{MODEL FITTING}

As a first test of the quality of the visibility data, we performed least square model fits to the visibility amplitudes and closure phases. The model included the positions and diameters of the stars as well as their intensity ratio. Uniformly bright disks were assumed for the model.

Table 1. Fitting Results

\begin{tabular}{cccccccc}
\hline UT Date & Intensity Ratio & \multicolumn{2}{c}{ Fitted Diam. } & \multicolumn{2}{c}{ Fitted Position $^{1}$} & \multicolumn{2}{c}{ Ref. Pos. $^{1,2}$} \\
& $I_{A a} / I_{A b}$ & $D_{A a}$ & $D_{A b}$ & dRA [mas] & dDEC [mas] & dRA & dDEC \\
\hline \hline $11 / 12 / 02$ & $1.39_{-0.67}^{+0.51}$ & $8.4 \pm 1.7$ & $5.6 \pm 2.5$ & $-9.61 \pm 0.84$ & $44.68 \pm 0.54$ & -8.93 & 44.70 \\
$11 / 13 / 02$ & $1.46_{-0.62}^{+0.50}$ & $8.5 \pm 1.1$ & $6.5 \pm 1.4$ & $-12.80 \pm 0.56$ & $43.96 \pm 0.29$ & -11.67 & 43.25 \\
$11 / 14 / 02$ & $1.34_{-0.64}^{+0.44}$ & $8.3 \pm 0.9$ & $5.8 \pm 1.4$ & $-13.89 \pm 0.30$ & $41.19 \pm 0.18$ & -14.47 & 41.60 \\
$11 / 15 / 02$ & $1.99_{-0.53}^{+0.51}$ & $8.0 \pm 2.9$ & $8.0 \pm 4.8$ & $-17.55 \pm 2.96$ & $40.02 \pm 0.98$ & -17.33 & 39.72 \\
$11 / 16 / 02$ & $1.69_{-0.38}^{+0.56}$ & $8.4 \pm 0.8$ & $7.2 \pm 2.0$ & $-20.14 \pm 3.25$ & $37.53 \pm 1.81$ & -20.13 & 37.68 \\
\hline
\end{tabular}

Notes:

1 Relative positions are measured from the infrared brighter to the infrared fainter component.

2 Reference orbit by Hummel et al.. ${ }^{6}$

The fit results for each individual night are given in Table 1, and Figure 1 shows the fitted model compared to the data for each night. The model fit generally agrees well with the data, and the measurements of the star 
positions are in good agreement with the high precision reference orbit obtained by Hummel et al. ${ }^{6}$ using the Mark III interferometer. These results give confidence in the procedures used to estimate visibilities and closure phases. Moreover, interesting new results and estimates may be obtained from the fits. Weighted averaging of the results for all nights gives, for the diameters of the Capella giants, $D_{A a}=8.4 \pm 0.2$ mas and $D_{A b}=6.3 \pm 0.7$ mas and the intensity ratio in the $\mathrm{H}$ band: $I_{A a} / I_{A b}=1.69 \pm 0.22$. Using Hipparcos parallaxes we calculate physical radii of $R_{A a}=11.7 \pm 0.3 R_{\odot}$ and $R_{A b}=8.8 \pm 1.0 R_{\odot}$.

\section{IMAGING}

The key goal of our project was to produce aperture synthesis images of Capella in order to demonstrate the capabilities of IOTA. However, observations were obtained over five days, during which time the relative positions of the binary pair changed by many milliarcsec. In order to make a single map with data from all nights, and thereby improve the UV coverage available for the map, we applied a coordinate transformation to compensate for the orbital motion of the binary components over the observed time interval. Thus, the actual UV points were transformed to a common system corresponding to the position of the stars at the middle of our observation run. Of course, this procedure is only strictly correct for certain models of the stellar system. For Capella, we feel that these special circumstances are somewhat justified since the cooler Aa component rotates synchronously to the orbital motion. The final UV coverage for the experiment, before the coordinate system transformation, is shown in Figure 2.

Many algorithms have been developed to reconstruct images from visibility data using the additional phase information provided by a closure phase measurement. Two of the most commonly used and notable methods are the conventional hybrid mapping approach ${ }^{7}(\mathrm{CHM})$ and the difference mapping approach ${ }^{8}$ (DFM). The main difference between these two approaches is that, whereas DFM starts with a specific initial model (in this case a binary) the CHM algorithm converges for simple brightness source distributions without any specific model. In this study, we have constructed images using both approaches. The necessary deconvolutions were performed with the classical CLEAN algorithm. ${ }^{9}$

The resulting CHM and DFM maps are presented in Fig. 3. Both mapping strategies yield similar results with noise levels below $7 \%$. We have a slight preference for the CHM method, as it converges without trial model assumption. However, the sidelobe levels in the DFM map are at a lower level and the speed of convergence is better since one may start with a model that is close to reality. The stellar surfaces are partially resolved in the map. We note, however, that the infrared-brighter Ab component rotates asynchronously to the orbital motion, ${ }^{10}$ and therefore, it would not be appropriate to interpret any observed structure as a real feature of that star.

Finally, as a test of the accuracy of the map, a deconvolution of the star images within the map was performed using the elliptical clean beam. The deconvolution confirmed independently our least squares fitting results for the intensity ratio and the diameters, within the errors. Therefore, we believe that the maps present an accurate picture of this binary star system.

\section{CONCLUSIONS}

By observing the well-studied Capella system we have been able to demonstrate the overall performance and imaging capabilities of the IOTA instrument. IOTA can successfully measure visibility amplitudes and closure phases, and this information can be used to construct images of simple sources. Thus, the primary goal of the program to upgrade IOTA to three telescopes has been achieved.

\section{ACKNOWLEDGMENTS}

We gratefully acknowledge the contributions of our many colleagues in the IOTA collaboration, including W. Traub, N. Carleton, M. Lacasse, M. Pearlman, E. Pedretti, J. Monnier, R. Millan-Gabet, and J.P. Berger. This work was supported by NSF grant AST-0138303. 


\section{REFERENCES}

1. W. A. Traub, A. Ahearn, N. P. Carleton, J. Berger, M. K. Brewer, K. Hofmann, P. Y. Kern, M. G. Lacasse, F. Malbet, R. Millan-Gabet, J. D. Monnier, K. Ohnaka, E. Pedretti, S. Ragland, F. P. Schloerb, K. Souccar, and G. Weigelt, "New Beam-Combination Techniques at IOTA," in Interferometry for Optical Astronomy II. Edited by Wesley A. Traub. Proceedings of the SPIE, Volume 4838, pp. 45-52 (2003)., pp. 45-52, Feb. 2003.

2. S. Kraus, M.S. thesis, University of Massachusetts at Amherst, 2003.

3. J. Berger, P. Haguenauer, P. Y. Kern, K. Rousselet-Perraut, F. Malbet, S. Gluck, L. Lagny, I. SchanenDuport, E. Laurent, A. Delboulbe, E. Tatulli, W. A. Traub, N. Carleton, R. Millan-Gabet, J. D. Monnier, E. Pedretti, and S. Ragland, "An integrated-optics 3-way beam combiner for IOTA," in Interferometry for Optical Astronomy II. Edited by Wesley A. Traub . Proceedings of the SPIE, Volume 4838, pp. 1099-1106 (2003)., pp. 1099-1106, Feb. 2003.

4. E. Pedretti, R. Millan-Gabet, J. D. Monnier, W. A. Traub, N. P. Carleton, J.-P. Berger, M. G. Lacasse, F. P. Schloerb, and M. K. Brewer, "The PICNIC Interferometry Camera at IOTA," Publications of the Astronomical Society of the Pacific 116, pp. 377-389, Apr. 2004.

5. J. D. Monnier, "Optical interferometry in astronomy," Reports of Progress in Physics 66, pp. 789-857, May 2003.

6. C. A. Hummel, J. T. Armstrong, A. Quirrenbach, D. F. Buscher, D. Mozurkewich, N. M. Elias, and R. E. Wilson, "Very high precision orbit of Capella by long baseline interferometry," Astronomical Journal 107, pp. 1859-1867, May 1994.

7. E. B. Fomalont and M. C. H. Wright, in Galactic and extra-galactic radio astronomy, Springer, 1974.

8. T. J. Pearson and A. C. S. Readhead, "Image Formation by Self-Calibration in Radio Astronomy," Annual Review of Astronomy and Astrophysics 22, pp. 97-130, 1984.

9. J. A. Högbom, "Aperture Synthesis with a Non-Regular Distribution of Interferometer Baselines," Astronomy and Astrophysics Supplement Series 15, pp. 417-+, June 1974.

10. K. G. Strassmeier, P. Reegen, and T. Granzer, "On the rotation period of Capella," Astronomische Nachrichten 322, pp. 115-124, Mar. 2001. 

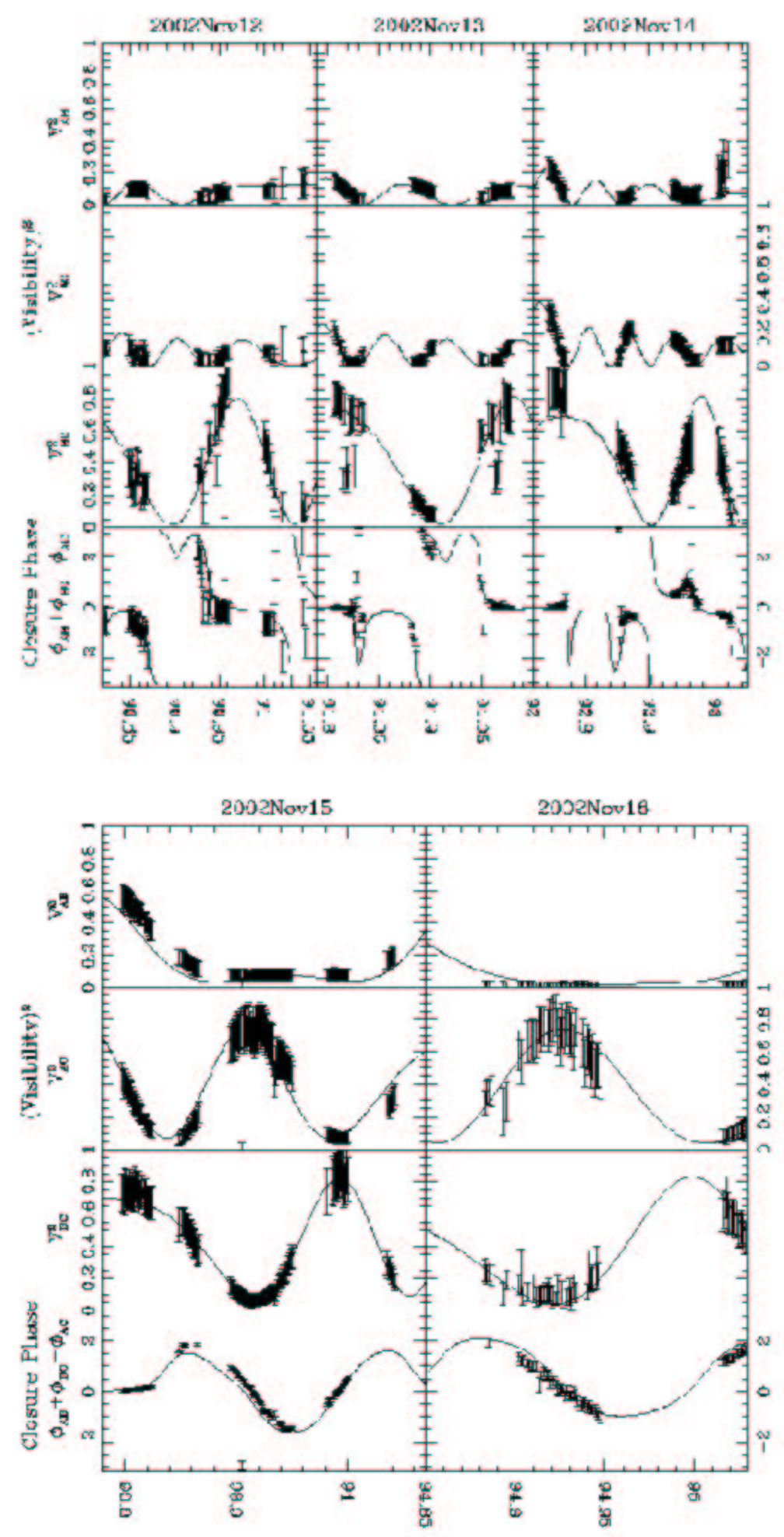

Figure 1. Best-fit models for Capella assuming uniform disks with parameters as listed in Table 1. Error bars shown on the data points reflect statistical errors and our estimate of the calibration errors. The abscissa depicts the date of the observation represented as MJD=JD-2452500. The square of the visibility amplitudes are presented for each baseline, and the Closure Phase for the three baselines, given in radians, is also presented. 


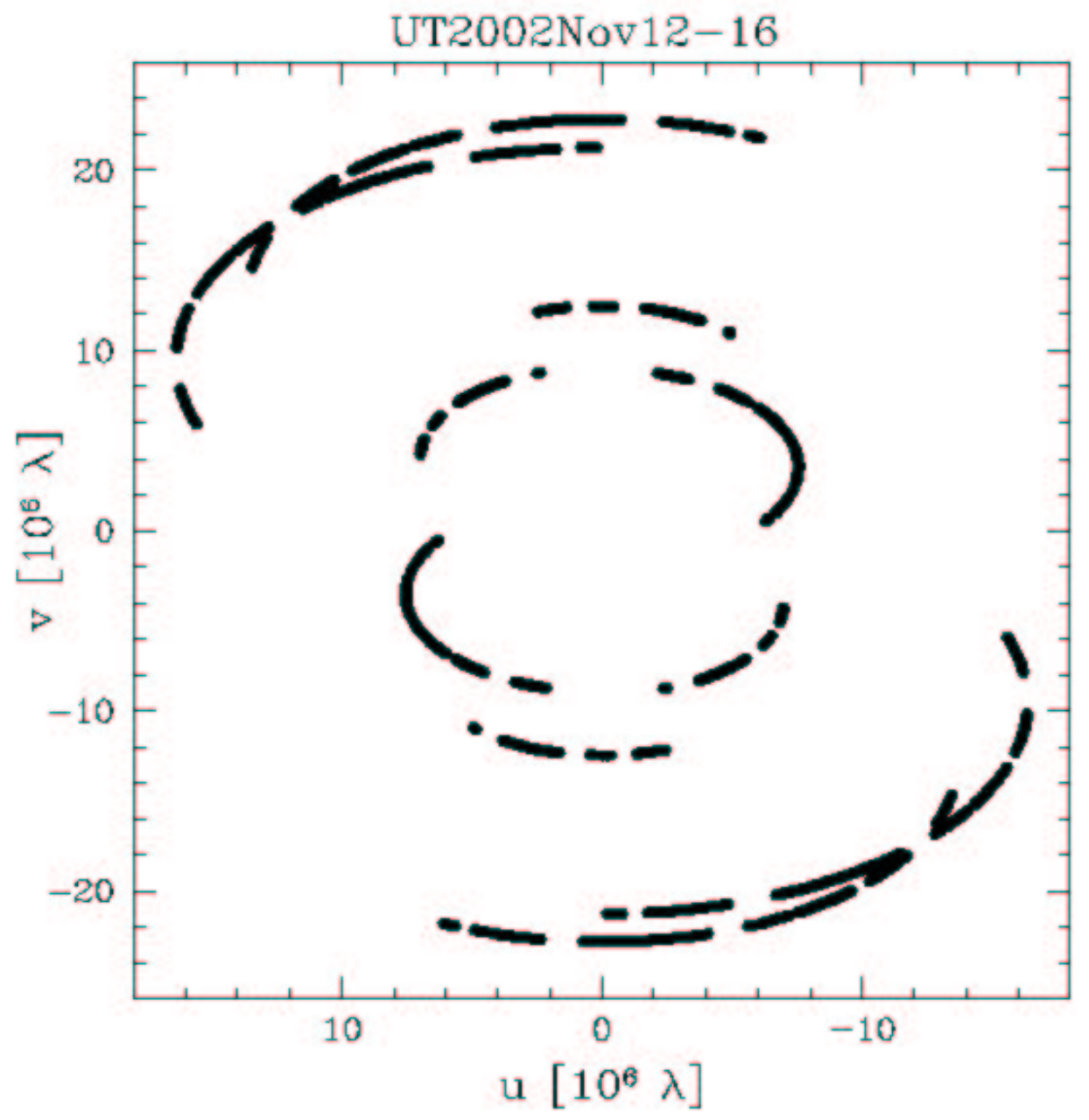

Figure 2. UV plane coverage for Capella observations made at the IOTA. For creating the final maps shown in Figure 3, each UV point is corrected for orbital motion of the binary stars in order to align the data from the five nights of observation. 

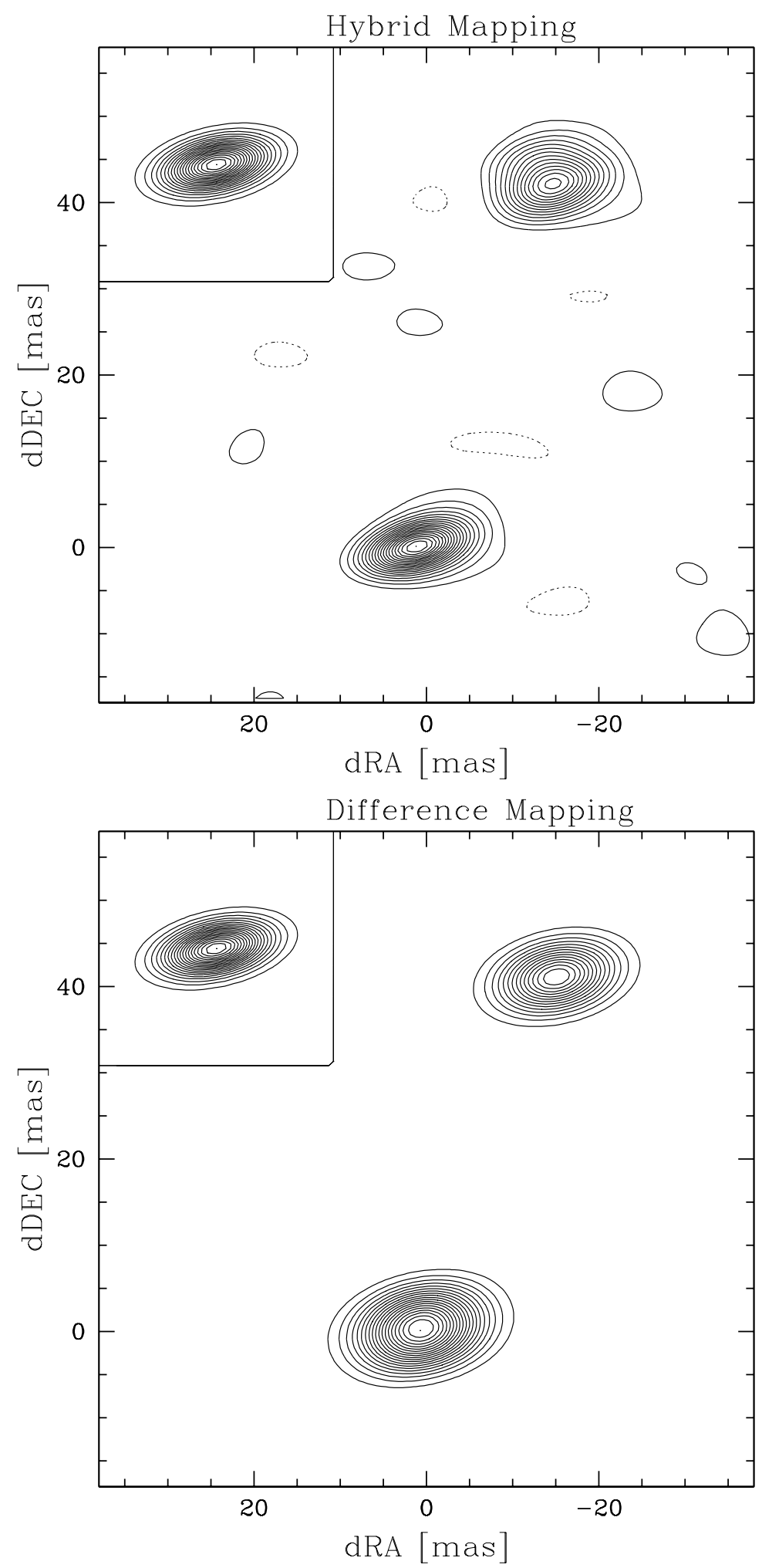

Figure 3. Capella aperture synthesis maps made by the conventional hybrid mapping approach, which does not make assumptions about the structure of the source (top), and the difference mapping approach, which relies on a model of the source to help convergence (bottom). Surface brightness is plotted with $5 \%$ interval contours (scaled to the peak intensity). The beam $(5.4 \times 2.6$ mas $)$ depicted in the upper-left corner. The maps are oriented north up, east left. 